MATHEMATICS OF COMPUTATION

Volume 78, Number 266, April 2009, Pages 1073-1087

S 0025-5718(08)02166-2

Article electronically published on November 20, 2008

\title{
COMPUTATION OF JACOBSTHAL'S FUNCTION $h(n)$ FOR $n<50$.
}

\author{
THOMAS R. HAGEDORN
}

\begin{abstract}
Let $j(n)$ denote the smallest positive integer $m$ such that every sequence of $m$ consecutive integers contains an integer prime to $n$. Let $P_{n}$ be the product of the first $n$ primes and define $h(n)=j\left(P_{n}\right)$. Presently, $h(n)$ is only known for $n \leq 24$. In this paper, we describe an algorithm that enabled the calculation of $h(n)$ for $n<50$.
\end{abstract}

0.1 . Introduction. Let $n$ be a positive integer. Every sequence of $n$ consecutive integers contains an $a$ with $(a, n)=1$. In [7, Jacobsthal raised the question: For a given $n$, what is the smallest number $m$ with the property that every sequence of $m$ consecutive integers contains an $a$ with $(a, n)=1$ ? The Jacobsthal function $j(n)$ is defined to be the smallest $m$ with this property. Equivalently, it is the largest difference between consecutive terms in the sequence of integers relatively prime to $n$. For example, we have $j(6)=4, j(30)=6$. Trivially, one has $j(n) \leq n$ and Jacobsthal conjectured

$$
j(n) \ll\left(\frac{\log n}{\log _{2} n}\right)^{2},
$$

where $f(x) \ll g(x)$ is understood to mean that there is a constant $C$ such that $|f(x)| \leq C g(x)$ for all $x$, and $\log _{k} x$ is the iterated logarithm defined by

$$
\log _{k} x= \begin{cases}\log x, & \text { if } k=1, \\ \log \left(\log _{k-1} x\right), & \text { if } k>1 .\end{cases}
$$

The best known upper bound,

$$
j(n) \ll \log ^{2} n,
$$

is due to Iwaniec [6].

If $m, n$ are both divisible by the same primes, then $j(m)=j(n)$. Hence, in studying $j(n)$, we can restrict our attention to $n$ that are the product of distinct primes. In this paper, we consider the particular case when $n$ is the product of the first $k$ primes. Let $P_{n}$ denote the product of the first $n$ primes and define

$$
h(n)=j\left(P_{n}\right) .
$$

Jacobsthal [7, Kanold [9, and Stevens [15] established upper bounds for $h(n)$; Maier and Pomerance [11] and Pintz [12] have established lower bounds for $h(n)$. Exact values for $h(n)$ have been previously calculated for $n \leq 24[8$ and are the

Received by the editor October 9, 2007 and, in revised form, March 23, 2008.

2000 Mathematics Subject Classification. Primary 11N25, 11Y55.

Key words and phrases. Jacobsthal function, killing sieve. 
TABLE 1 . Values of $h(n)$ for $n \leq 49$

\begin{tabular}{r|rr|rr|rr|rr|r}
$n$ & $h(n)$ & $n$ & $h(n)$ & $n$ & $h(n)$ & $n$ & $h(n)$ & $n$ & $h(n)$ \\
\hline 1 & 2 & 11 & 58 & 21 & 190 & 31 & 354 & 41 & 550 \\
2 & 4 & 12 & 66 & 22 & 200 & 32 & 378 & 42 & 574 \\
3 & 6 & 13 & 74 & 23 & 216 & 33 & 388 & 43 & 600 \\
4 & 10 & 14 & 90 & 24 & 234 & 34 & 414 & 44 & 616 \\
5 & 14 & 15 & 100 & 25 & 258 & 35 & 432 & 45 & 642 \\
6 & 22 & 16 & 106 & 26 & 264 & 36 & 450 & 46 & 660 \\
7 & 26 & 17 & 118 & 27 & 282 & 37 & 476 & 47 & 686 \\
8 & 34 & 18 & 132 & 28 & 300 & 38 & 492 & 48 & 718 \\
9 & 40 & 19 & 152 & 29 & 312 & 39 & 510 & 49 & 742 \\
10 & 46 & 20 & 174 & 30 & 330 & 40 & 538 & &
\end{tabular}

unshaded entries in Table 1. In this paper, we present an algorithm that enabled us to calculate $h(n)$ for $25 \leq k \leq 49$. These values appear as the shaded entries in Table 1. In Figure 1, we graph the exact values for $h(n)$ versus $A(n)$ (see (1.4)), the main term of the best known asymptotic lower bound for $h(n)$. In Figure 2, we graph the ratio $h(n) / A(n)$.

In Section 1 we review the upper and lower bounds that have been established for $h(n)$ and present the connection between $h(n)$ and the problem of determining large gaps between consecutive primes. In Section 2, we introduce killing sieves and relate them to $h(n)$. In Section 3 we describe a bound that reduces the search space for finding a maximal $\left(S_{n}, k\right)$-killing sieve. In Section 4 we describe the algorithm that permits an efficient calculation of $h(n)$ using killing sieves. Upon first reading this section, one should follow the example in Section 5. In Section 6, we discuss our use of distributed computing and the technical details of our computation. At the end of the paper, we include data tables that determine a sequence of $h(n)-1$ consecutive integers, each of which is divisible by one of the first $n$ primes.

Notation. Finally, we list the following notations that are used throughout the paper: $[1, z]=$ the set of positive integers $\leq z$.

$p_{i}$ denotes the $i$ th prime.

$q_{i}$ denotes the $i$ th odd prime.

$S_{n}=\left\{q_{1}, \ldots, q_{n}\right\}$ is the set of the first $n$ odd primes.

$P(x)$ is the product of the primes $p \leq x$.

$P_{n}$ is the product of the first $n$ primes.

\section{Bounds ON $h(n)$}

Though exact values for $h(n)$ are difficult to compute, there has been extensive work done on establishing upper and lower bounds for $h(n)$. Previous to the estimate in (0.1), Iwaniec [5] showed

$$
h(n) \ll n^{2} \log ^{2} n .
$$

Iwaniec's bound is proved using sieve theory. Using very elementary arguments, Kanold 9] proved

$$
h(n) \leq 2^{n}
$$




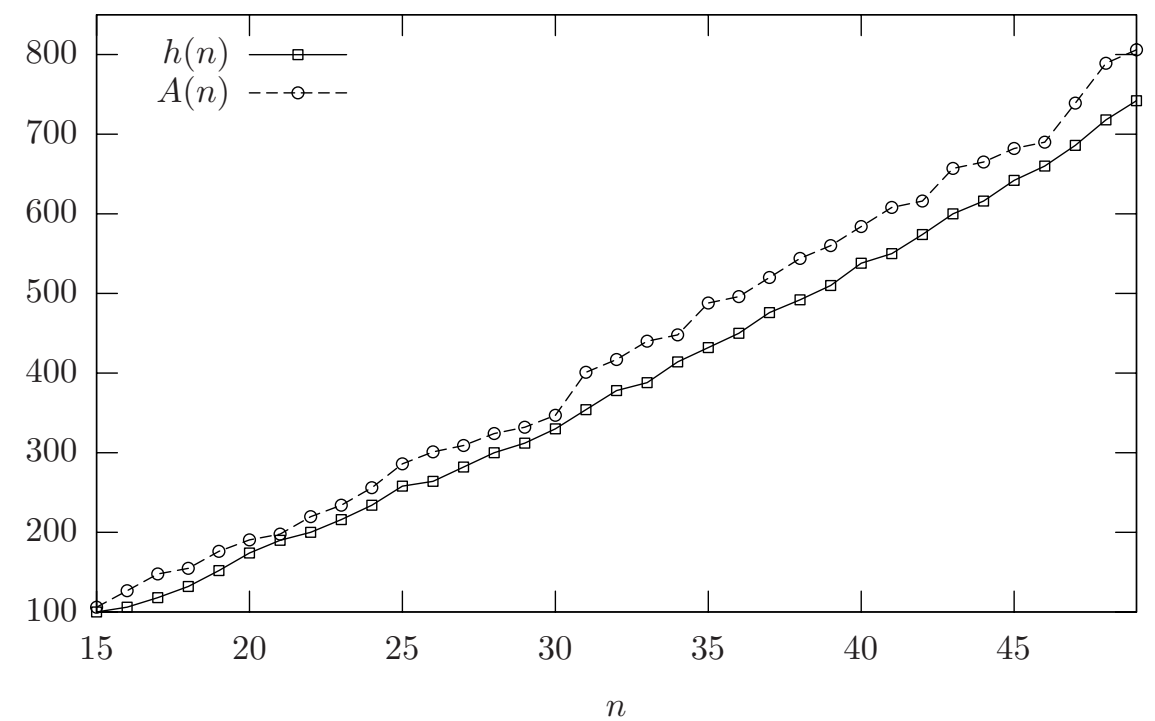

Figure 1. Comparison of $h(n)$ with the asymptotic lower bound $A(n)$

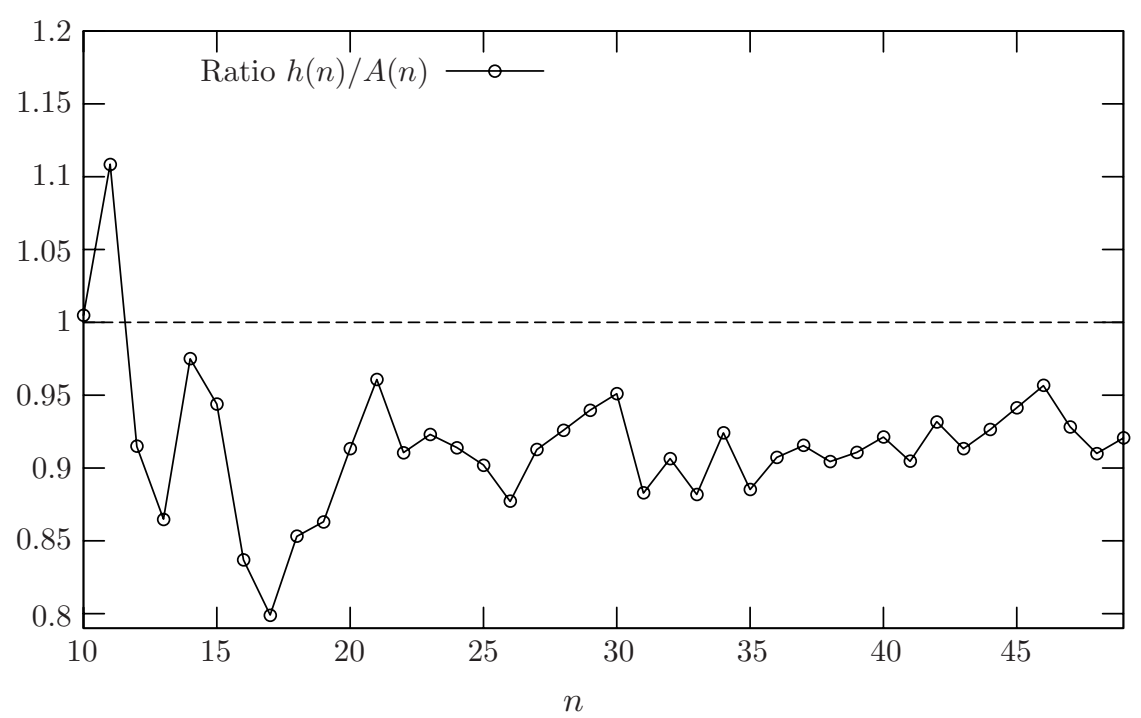

FiguRE 2. Ratio of $h(n)$ to the asymptotic lower bound $A(n)$

for all $n$ and $h(n) \leq 2^{\sqrt{n}}$, if $n \geq e^{50}$. By elementary means, Stevens [15] proved for $n \geq 15$ the stronger (for $n>4,000,000$ ) bound

$$
h(n) \leq 2 n^{2+2 e \log n} .
$$

While considerably weaker asymptotically than (1.1), (1.2) provides an explicit constant.

The best lower bound for $h(n)$ is due to Pintz [12, improving on previous work of Maier and Pomerance [11. Define $P(x)$ to be the product of the primes less 
than or equal to $x$. Pintz proved

$$
j(P(x)) \geq\left(2 e^{\gamma}+o(1)\right) \frac{x \log x \log _{3} x}{\log _{2}^{2} x},
$$

where $\gamma \approx .577216$ is Euler's constant. Letting $x=p_{n}$, the $n$th prime, we obtain

$$
h(n) \geq\left(2 e^{\gamma}+o(1)\right) \frac{p_{n} \log p_{n} \log _{3} p_{n}}{\log _{2}^{2} p_{n}} .
$$

We define

$$
A(n)=2 e^{\gamma} \frac{p_{n} \log p_{n} \log _{3} p_{n}}{\log _{2}^{2} p_{n}} .
$$

$A(n)$ represents the asymptotic lower bound function for $h(n)$. We compare its values with those of $h(n)$ in Figures 1 and 2 .

Let $J(x)=\max _{n \leq x} j(n)$. Combining (1.3) with the approximation $P(\log x) \approx x$, [11, 12 established a similar lower bound for $J(x)$. [12 showed that

$$
J(x) \geq\left(2 e^{\gamma}+o(1)\right) \frac{\log x \log _{2} x \log _{4} x}{\log _{3}^{2} x} .
$$

For completeness, we mention that Maier and Pomerance have conjectured that

$$
J(x)=O\left(\log x\left(\log _{2} x\right)^{2+o(1)}\right) .
$$

Lastly, we note that estimate (1.5) is then used by [11, 12, to establish the same lower bound as in (1.5) for the function

$$
G(x)=\max _{p_{n} \leq x}\left(p_{n+1}-p_{n}\right),
$$

which measures the maximal gap between two consecutive primes with the smaller prime $\leq x$. This connection with the maximal gaps between consecutive primes is a major motivation for the study of lower bounds for the Jacobsthal function.

The lower bounds for $h(n)$ established above are all based on sieve methods. In contrast, the strongest result proved algebraically without sieve methods is the much weaker result:

Proposition 1.1. For $n>1, h(n) \geq 2 p_{n-1}$.

Proof. Let $N=p_{1} \cdots p_{n-2}$ and $\epsilon= \pm 1$. By the Chinese Remainder Theorem, we can find an integer $x$ such that

$$
x \equiv 0 \bmod N, \quad x \equiv \epsilon \bmod p_{n-1}, \quad \text { and } \quad x \equiv-\epsilon \bmod p_{n} .
$$

Then $x-p_{n-1}, x+p_{n-1}$ are consecutive terms in $\mathbb{Z}_{p_{1} \ldots p_{n}}^{*}$ with a gap of length $2 p_{n-1}$.

\section{Killing Sieves}

We use the same notations as described at the end of the Introduction. Because $P_{n}$, the product of the first $n$ primes, grows exponentially as a function of $n$, it is impractical to determine $h(n)$ for $n>20$ by a brute-force search of the gaps between elements of $\mathbb{Z}_{P_{n}}^{*}$. To compute $h(n)$ for $n<50$, we employ a reduction, based on a generalization of an idea of J. Haugland [4, that uses killing sieves. We note that algorithms similar in spirit were used by Gordon and Rodemich 3 to study admissible sets for the Prime $k$-tuples Conjecture. In this section, we define killing sieves and relate them to the function $h(n)$. 
Definition 2.1. Let $r \geq k \geq 0$ be integers and let $S=\left\{t_{1}, \ldots, t_{r}\right\}$ be a set of primes. An $S$-sieve with $k$ elements is a set $T$, where

$$
T=\left\{\left(i_{1}, c_{1}\right), \ldots,\left(i_{k}, c_{k}\right)\right\}, \text { with integers } 1 \leq i_{1}<i_{2}<\ldots<i_{k} \leq r,
$$

and $c_{j} \in \mathbb{Z}_{t_{i_{j}}}$ is an equivalence class $\bmod t_{i_{j}}$, for $j=1, \ldots, k$.

Definition 2.2. Let $r \geq k \geq 0$ be integers, let $S=\left\{t_{1}, \ldots, t_{r}\right\}$ be a set of primes, and let $z \geq 1$. Let $T=\left\{\left(i_{j}, c_{j}\right)\right\}_{1 \leq j \leq r-k}$ be an $S$-sieve with $r-k$ elements. $T$ is called an $(S, k)$-killing sieve of length $z$ if there exists a set $I \subset[1, z]$ of $k$ distinct integers with the property that for all $x \in[1, z] \backslash I$, there exists $j \in[1, r-k]$ such that $x \equiv c_{j} \bmod t_{i_{j}}$. We say that an $(S, k)$-killing sieve $T$ has maximal length $z$ if there is no $(S, k)$-killing sieve with length greater than $z$.

Remarks 2.3. (i) If $T$ is an $(S, 0)$-killing sieve of length $z$, for simplicity we refer to it as a $S$-killing sieve of length $z$.

(ii) If $S$ is the set of the first $n$ primes, then an $S$-killing sieve of maximal length has length $h(n)-1$.

(iii) If $T^{\prime} \subset T$, where $T$ is an $(S, k)$-killing sieve of length $z$, we say $T^{\prime}$ can be extended to a $(S, k)$-killing sieve of length $z$.

(iv) By the Chinese Remainder Theorem, we can assume that there is an integer $c$ such that all (or any subset) of the $c_{j}$ in the definition of $T$ satisfy $c_{j} \equiv$ $c \bmod t_{i_{j}}$.

Examples 2.4. (i) Let $S=\{2,3,5\}=\left\{t_{1}, t_{2}, t_{3}\right\}$. Then $T=\{(1,1),(2,2),(3,4)\}$ is an $S$-killing sieve of length 5 as $x=1,3,5 \in[1,5]$ satisfy $x \equiv 1 \bmod t_{1}$, $x=2$ satisfies $x \equiv 2 \bmod t_{2}$, and $x=4$ satisfies $x \equiv 4 \bmod t_{3}$. The following table illustrates the smallest prime $t \in S$ used for each $x \in[1,5]$ :

\begin{tabular}{|l|l|l|l|l|l|}
\hline$x$ & 1 & 2 & 3 & 4 & 5 \\
\hline$t$ & 2 & 3 & 2 & 5 & 2 \\
\hline
\end{tabular}

(ii) Let $S=\{2\}$. Then $T=\{(1,1)\}$ is an $(S, 2)$-killing sieve of length 5 , as there are only two integers $x \in[1,5]$ that do not satisfy $x \equiv 1 \bmod 2$. The corresponding table is:

\begin{tabular}{|l|l|l|l|l|l|}
\hline$x$ & 1 & 2 & 3 & 4 & 5 \\
\hline$t$ & 2 & $*$ & 2 & $*$ & 2 \\
\hline
\end{tabular}

where the $*$ indicates that the corresponding $x$ is in the set $I$ associated to $T$.

(iii) Let $S_{8}=\{3,5,7,11,13,17,19,23\}$. Then

$$
T=\{(1,2),(2,7),(3,6),(4,4),(5,3),(6,1)\}
$$

defines an $\left(S_{8}, 2\right)$-killing sieve of length 18 . Its associated table is:

\begin{tabular}{|c|c|c|c|c|c|c|c|c|c|c|c|c|c|c|c|c|c|c|}
\hline$x$ & 1 & 2 & 3 & 4 & 5 & 6 & 7 & 8 & 9 & 10 & 11 & 12 & 13 & 14 & 15 & 16 & 17 & 18 \\
\hline$q$ & 17 & 3 & 13 & 11 & 3 & 7 & 5 & 3 & $*$ & $*$ & 3 & 5 & 7 & 3 & 11 & 13 & 3 & 17 \\
\hline
\end{tabular}

(iv) Let $S_{19}=\{3,5, \ldots, 71\}$ be the set of the first 19 odd prime numbers. Then

$$
\begin{aligned}
T=\{ & (1,2),(2,4),(3,1),(4,7),(5,3),(6,10),(7,6),(8,14), \\
& (9,12),(10,21),(11,30),(12,31),(13,33),(14,28),(15,13)\}
\end{aligned}
$$

(the second entries come from the column for $n=19$ in Table 3) is an $\left(S_{19}, 4\right)$ killing sieve of length 86 . Except for $x=45,46,48,58$, all $x \in[1,86]$ satisfy 
$x \equiv c_{j} \bmod t_{i_{j}}$ for some $j$. Letting

$$
T^{\prime}=T \cup\{(16,45),(17,46),(18,48),(19,58)\}
$$

we obtain an $S_{19}$-killing sieve of length 86 . The additional elements of $T^{\prime}$ indicate that the primes $t_{16}=59, t_{17}=61, t_{18}=67, t_{19}=71$ each eliminate exactly one integer from $[1, z]$. These are the integers $\{45,46,48,58\}$ that do not satisfy the congruence criteria of the other primes $t_{j} . T^{\prime}$ is not unique as there are $4 !=24$ different $S_{19}$-killing sieves $T^{\prime}$ that arise from $T$.

Proposition 2.5. Let $S$ be a set of $r$ distinct primes. There is an $S$-killing sieve of length $z$ if and only if there is an $(S, k)$-killing sieve of length $z$ for some $k \in[0, r]$.

Proof. $(\Rightarrow)$ is clear since we can take $k=0$. $(\Leftarrow)$ Let $T$ be an $(S, k)$-killing sieve of length $z$ and let $I=\left\{c_{1}, \ldots, c_{k}\right\}$ be the associated set of $k$ distinct integers in $[1, z]$. Let $j_{1}, \ldots, j_{k}$ be the $k$ integers in $[1, r]$ that do not appear as a first element of the pairs in $T$ (see (2.1) ). Then

$$
T^{\prime}=T \cup\left\{\left(j_{1}, c_{1}\right), \ldots,\left(j_{k}, c_{k}\right)\right\}
$$

is an $(S, 0)$-killing sieve of length $z$.

It is useful to reduce the problem of searching for an $(S, k)$-killing sieve to the problem of searching for an $(S, k+1)$-killing sieve. The following elementary lemma gives a specific case when we can do this reduction.

Lemma 2.6. Let $S=\left\{t_{1}, \ldots, t_{n}\right\}$ be a set of $n$ distinct primes. Let $T$ be an $(S, n-r)$-killing sieve of length $z$, and let $T=\left\{\left(i_{j}, c_{j}\right)\right\}_{1 \leq j \leq r}$. Suppose that there is $j_{0} \in[1, r]$ and $y \in[1, z]$ with the property that $y \equiv c_{j_{0}} \bmod t_{i_{j_{0}}}$ and $y \neq \equiv c_{j} \bmod t_{i_{j}}$, for $j \neq j_{0}$. Then $\hat{T}=T \backslash\left(i_{j_{0}}, c_{j_{0}}\right)$ is an $(S, n-r+1)$-killing sieve of length $z$ with the property that no pair in $\hat{T}$ has $i_{j_{0}}$ as its first coordinate.

Proof. If $I \subset[1, z]$ is the set of $k$ elements associated with $T$, let $\hat{I}=I \cup\left\{y_{0}\right\}$ be the set associated to $\hat{T}$. The lemma follows immediately.

We now define the function $w(n)$ and relate it to the function $h(n)$.

Definition 2.7. For $n \geq 1$, we define $w(n)$ to be the maximal length of an $S_{n^{-}}$ killing sieve, where $S_{n}$ is the set of the first $n$ odd primes.

Proposition 2.8. For $n \geq 1, h(n+1)=2 w(n)+2$.

Proof. Let $h=h(n+1)$. We first show that $h \leq 2 w(n)+2$. By definition of $h$, there is an integer $b$ such that each term in the sequence

$$
b+1, b+2, \ldots, b+h-1
$$

is divisible by one of the first $n+1$ primes $p_{i}$. If $\left(b, P_{n+1}\right) \neq 1$ or $\left(b+h, P_{n+1}\right) \neq 1$, the sequence in (2.2) would then be a subsequence of a longer sequence with the same property. As this would imply $h(n+1)>h$, we must have $\left(b, P_{n+1}\right)=$ $\left(b+h, P_{n+1}\right)=1$. In particular, $b$ is odd and $h$ must be even. Thus, (2.2) gives an arithmetic sequence of odd integers

$$
b+2, b+4, \ldots, b+h-2,
$$

where each term is divisible by one of the odd primes $p_{2}, \ldots, p_{n+1}$. Let $N=$ $p_{2} \cdots p_{n+1}$ and choose $\beta \in \mathbb{Z}$ such that $2 \beta \equiv 1 \bmod N$. Then the sequence

$$
\beta(b+2), \beta(b+4), \ldots, \beta(b+h-2)
$$


derived from (2.3) is a sequence of $w=(h-2) / 2$ consecutive terms in $\mathbb{Z}_{N}$, each of which is a nonunit. Let $a=\beta(b+2)$. Then rewriting (2.4), each term of the sequence

$$
a+1, a+2, \ldots, a+w
$$

is divisible by one of the odd primes $p_{2}, \ldots, p_{n+1}$. Equivalently, for every integer $i \in[1, w]$, there exists $j \in\{2, \ldots, n+1\}$ such that

$$
i \equiv-a \bmod q_{j}
$$

Hence there is an $S_{n}$-killing sieve of length $w$. Since $w \leq w(n)$, we have $h=$ $2 w+2 \leq 2 w(n)+2$. Conversely, if there is an $S_{n}$-killing sieve of length $w(n)$, these steps can be reversed to show $h(n+1) \geq 2 w(n)+2$. The proposition then follows.

\section{Bounding A KILling SIEve}

In this section, we establish a bound in Proposition 3.10 that gives an efficient means to search through all possible $\left(S_{n}, k\right)$-killing sieves of maximal length. We use the same notations as described at the end of the Introduction and recall that $S_{n}=\left\{q_{1}, \ldots, q_{n}\right\}$ is the set of the first $n$ odd primes.

Let $n \geq 1$ and assume $T=\left\{\left(i_{1}, c_{1}\right), \ldots,\left(i_{r}, c_{r}\right)\right\}$ is an $S_{n}$-sieve for some $r \leq n$. For a positive integer $z$, define:

$$
I_{j}=I_{j}(T, z)= \begin{cases}{[1, z]} & \text { if } j=0, \\ \left\{x \in I_{j-1} \text { such that } x \not \equiv c_{j} \bmod q_{i_{j}}\right\} & \text { if } 1 \leq j \leq r \\ I_{r} & \text { if } j>r\end{cases}
$$

and define

$$
n_{j}= \begin{cases}\left|I_{j-1}\right|-\left|I_{j}\right|, & \text { if } 1 \leq j \leq r \\ 0, & \text { otherwise }\end{cases}
$$

The number $n_{j}$ represents the number of integers in $I_{j-1}$ that are in the congruence class $c_{j} \bmod q_{i_{j}}$. By definition, $z \geq \sum_{j=1}^{r} n_{j}$. We can then use the sum to determine when $T$ is an $\left(S_{n}, n-r\right)$-sieve.

Lemma 3.1. Let $T, z$, and $n_{j}$ be defined as above. Then $T$ is an $\left(S_{n}, n-r\right)$-killing sieve of length $z$ if and only if $z \leq(n-r)+\sum_{j=1}^{r} n_{j}$.

Proof. $(\Rightarrow)$ is clear. $(\Leftarrow)$ We have $\left|I_{r}\right|=z-\sum_{j=1}^{r} n_{j} \leq n-r$ by hypothesis. Then $T$ and $I_{r}$ together define an $\left(S_{n}, n-r\right)$-killing sieve.

We now prove a useful upper bound for the $n_{j}$. The case $i=1$ is due to J. Haugland [4.

Definition 3.2. Let $\left\{m_{i}\right\}_{i \geq 0}$ be the sequence of positive integers defined by $m_{0}=$ $1, m_{1}=3, m_{2}=4, m_{3}=6, m_{4}=8$, and $m_{i}=10$ for $i \geq 5$. 
Proposition 3.3. Assume that $T$ is an $\left(S_{n}, k\right)$-killing sieve of length $z$ with $i_{1}=1$, $i_{2}=2$ (as defined in (2.1)) and that

(i) $i \in\{1,2,3\}$ and $j>1$, or

(ii) $i \in\{4,5\}$ and $j>2$.

If $m_{i} q_{i_{j}}>z-1$, then $n_{j} \leq i+1$.

Proof. (i) We first let $i=1$. Suppose $n_{j}>2$ and let $y \in[1, z]$ be the smallest integer in $I_{j-1}-I_{j}$. Since $j>1, y \in I_{1}$ and $y \not \equiv c_{1} \bmod 3$. Since $n_{j}>2$, there are positive integers $t_{2}>t_{1}$ such that $\left\{y, y+t_{1} q_{i_{j}}, y+t_{2} q_{i_{j}}\right\} \subset I_{j-1} \subset I_{1}$. However, since either $y+q_{i_{j}}$ or $y+2 q_{i_{j}}$ is congruent to $c_{1} \bmod 3$, one of these elements is not in $I_{1}$. Hence $t_{2} \geq 3$ and

$$
3 q_{i_{j}} \leq t_{2} q_{i_{j}}=\left(y+t_{2} q_{i_{j}}\right)-y \leq z-1,
$$

which contradicts the hypothesis. The cases when $i=2,3$ are proved similarly. We now prove (ii). Let $i=5$ and $j>2$. Let $y$ be the smallest element in $I_{j-1}-I_{j}$. Assume $n_{j}>6$. Then there are positive integers $t_{6}>t_{5}>\cdots>t_{1}$ such that

$$
S=\left\{y, y+t_{1} q_{i_{j}}, \ldots, y+t_{6} q_{i_{j}}\right\} \subset I_{j-1} \subset I_{2} .
$$

Either $y+q_{i_{j}}$ or $y+2 q_{i_{j}}$ is congruent to $c_{1} \bmod 3$. Suppose $y+2 q_{i_{j}} \equiv c_{1} \bmod 3$. Then $t_{k} \not \equiv 2 \bmod 3$ for each $k$ and we must have $t_{6} \geq 9$. If $t_{6}=9$, then $\left\{t_{1}, \ldots, t_{6}\right\}=$ $\{1,3,4,6,7,9\}$. However, with this set of $t_{k}$, since $q_{i_{j}} \neq 5$, the set $S$ in (3.1) contains an element in each of the congruence classes $\{0,1,2,3,4\} \bmod 5$. This results in a contradiction as one of the terms $y+t_{k} q_{i_{j}}$ would not be an element of $I_{2}$. Hence $t_{6} \geq 10$ and from (3.1), we have

$$
10 q_{i_{j}} \leq t_{6} q_{i_{j}}=\left(y+t_{6} q_{i_{j}}\right)-y \leq z-1,
$$

which contradicts the hypothesis. An identical argument when $y+q_{i_{j}} \equiv c_{1} \bmod 3$ also gives a contradiction. Hence, the initial assumption is wrong and $n_{j} \leq 6$. A similar argument proves (ii) for $i=4$.

Remark 3.4. We note that the lemma can be generalized to $i>5$, but these cases have not been useful for the calculation of $h(n)$.

Definition 3.5. Let $q, z$ be integers satisfying $(z-1)<10 q$. We define $m(q, z)=$ $1+i$, where $i$ is the smallest natural number such that $m_{i}>(z-1) / q$.

Definition 3.6. Define $S_{n}(z)$ to be the set of primes $q \in S_{n}$ with $10 q \leq z-1$. Define $r_{n}(z)=\left|S_{n}(z)\right|$ and $R_{n}(z)=\prod_{q \in S_{n}(z)} q$.

Example 3.7. Let $n=3, z=53$. Then $S_{3}(53)=\{3,5\}$. We have $S_{3} \backslash S_{3}(53)=$ $\{7\}$. Then $m(7,53)=1+4=5$ as $m_{4}=8>(53-1) / 7>m_{3}=6$. We have $r_{3}(53)=2$ and $R_{3}(53)=15$.

Later, we will need to use the following elementary lemma:

Lemma 3.8. Let $n, z$ be given.

(i) If $n \geq 3$ and $71 \leq z \leq 105$, then $z \leq R_{n}(z)$.

(ii) If $n \geq 4$ and $111 \leq z \leq \prod_{i=1}^{n} q_{i}$, then $z \leq R_{n}(z)$. 
We now establish a fundamental bound that expedites the calculation of $h(n)$. We first make the following definition.

Definition 3.9. Let $n, z \geq 1$. For $k \geq r_{n}(z)$, define

$$
M(n, z, k)=\sum_{i=k+1}^{n} m\left(q_{i}, z\right)
$$

We note that $i \geq k+1>r_{n}(z)$ and thus the summand term $m\left(q_{i}, z\right)$ is defined.

Proposition 3.10. Let $n, z \geq 1$ and assume $k \geq r_{n}(z)$. Let $T=\left\{\left(i_{j}, c_{j}\right)\right\}_{1 \leq j \leq k}$ be an $S_{n}$-sieve with $i_{j}=j$ for $j=1, \ldots, r_{n}(z)$. Assume that the elements of $T$ are the first $k$ pairs of $a\left(S_{n}, i_{k}-k\right)$-killing sieve of length $z$. Then

$$
\left|I_{k}(T)\right| \leq i_{k}-k+M\left(n, z, i_{k}\right)
$$

Proof. By definition, the $\left(S_{n}, i_{k}-k\right)$-killing sieve must consist of pairs $\left(i_{j}, c_{j}\right)$ for $j=1, \ldots, n+k-i_{k}$. By Lemma 3.1. we have $z \leq i_{k}-k+\sum_{j=1}^{n+k-i_{k}} n_{j}$. Since $\left|I_{k}\right|=z-\sum_{j=1}^{k} n_{j}$ and $n_{j} \leq m\left(q_{i_{j}}, z\right)$, we have:

$$
\left|I_{k}\right|-\left(i_{k}-k\right) \leq \sum_{j=k+1}^{n+k-i_{k}} n_{j} \leq \sum_{j=k+1}^{n+k-i_{k}} m\left(q_{i_{j}}, z\right) \leq \sum_{j=i_{k}+1}^{n} m\left(q_{j}, z\right)=M\left(n, z, i_{k}\right) .
$$

Thus 3.2 is proved.

\section{The Algorithm}

In this section, we describe an efficient algorithm for calculating $w(n)$ by finding an $S_{n}$-killing sieve of maximal length. We use the same notations as described at the end of the Introduction. Recall that $S_{n}=\left\{q_{1}, \ldots, q_{n}\right\}$ is the set of the first $n$ odd primes and that $r_{n}(z), R_{n}(z)$ are defined in Definition 3.6.

Assume $n \geq 4$. Suppose that $T=\left\{\left(i_{1}, c_{1}\right), \ldots,\left(i_{n}, c_{n}\right)\right\}$ is an $S_{n}$-killing sieve of length $z$. By Remark 2.3(iv), there is an integer $c$ with the property that the set $T^{\prime}=\left\{\left(1, c \bmod q_{1}\right), \ldots,\left(r_{n}(z), c \bmod q_{r_{n}(z)}\right)\right\}$ is a subset of $T$. We note that $I_{r_{n}(z)}(T)=I_{r_{n}(z)}\left(T^{\prime}\right)$ and denote it by $I_{r_{n}(z)}$.

The elements of $I_{r_{n}(z)}$ defined in (3.2) can then be identified with the units $\bmod R_{n}(z)$ in the sequence of integers $1-c, \ldots, z-c$. Additionally, since we are ultimately trying to find a maximal $S_{n}$-killing sieve, we can assume that $c$ is also a unit $\bmod R_{n}(z)$. Assume $111 \leq z \leq \prod_{i=1}^{n} q_{i}$ (in practice, the upper bound is always satisfied). By Lemma 3.8] we can assume $z \leq R_{n}(z)$ and the terms of the sequence will be distinct $\bmod R_{n}(z)$.

In a naive brute-force algorithm to find $T$, one tries all possible $c \in \mathbb{Z}_{R_{n}(z)}^{*}$ (which determines the subset $T^{\prime}$ ) and congruence classes $c_{j} \bmod q_{i_{j}}$, for $r_{n}(z) \leq j \leq n$ (which determines $T \backslash T^{\prime}$ ). However, this algorithm can be sharpened to permit a more efficient search. First, by Proposition 3.10, $I_{r_{n}(z)}$ must satisfy

$$
\left|I_{r_{n}(z)}\right| \leq M\left(n, z, r_{n}(z)\right) \text {. }
$$

Otherwise, $T^{\prime}$ cannot be extended to an $S_{n}$-killing sieve of length $z$. Hence, one must choose $c \in \mathbb{Z}_{R_{n}(z)}^{*}$ so that (4.1) is satisfied. Second, by Lemma 2.6, we can assume that $T$ is an $\left(S_{n}, n-r\right)$-killing sieve of length $z$ with $r_{n}(z) \leq r \leq n$ and $T^{\prime} \subset T$. Then

$$
T \backslash T^{\prime}=\left\{\left(i_{j}, c_{j}\right) \mid \text { where } r_{n}(z)+1 \leq j \leq r\right\},
$$


for some integers $i_{r_{n}(z)+1}, \ldots, i_{r}$ satisfying $r_{n}(z)<i_{r_{n}(z)+1}<\ldots<i_{r} \leq n$, and $c_{j} \bmod q_{i_{j}}$ for $r_{n}(z)+1 \leq j \leq r$. For each $k \in\left[r_{n}(z), r\right]$, we have

$$
\left|I_{k}(T)\right| \leq\left(i_{k}-k\right)+M\left(n, z, i_{k}\right),
$$

by Proposition 3.10. Most choices of $c, i_{j}$, and $c_{j} \bmod q_{i_{j}}$ do not satisfy (4.1) and (4.2), and can be discarded. Due to the reduced number of possible $c, i_{j}$, and congruence classes $c_{j}$ mod $q_{i_{j}}$ to consider, a modified brute-force search can successfully determine a maximal $\left(S_{n}, n-r\right)$-killing sieve for $n<49$, for some $r$.

We now discuss how $r$ and the $i_{j}$ for $j>r_{n}(z)$ are chosen. As there are fewer choices of parameters $c_{j}$ mod $q_{i_{j}}$ in an $\left(S_{n}, h+1\right)$-killing sieve as compared to an $\left(S_{n}, h\right)$-killing sieve, one would like to make this reduction whenever possible. We repeatedly make the reduction in the following case. Given an $\left(S_{n}, h\right)$-killing sieve $T$, and two integers $j, k$ with $0<k<j \leq n-h$, suppose that no two of the elements of $\left|I_{k}(T)\right|$ lie in the same congruence class $\bmod q_{i_{j}}$ (with $i_{j}$ as in (2.1)). Then $\left(i_{j}, c\right) \in T$ for some $c$. By Lemma 2.6, $\hat{T}=T \backslash\left(i_{j}, c\right)$ is an $\left(S_{n}, h+1\right)$-killing sieve in which $i_{j}$ does not appear as the first coordinate of any pair.

Combining these observations, we obtain an algorithm (detailed in Table 4) for determining the maximal length of an $S_{n}$-killing sieve. The algorithm begins by searching for an $S_{n}$-killing sieve of length $z$, where $z$ is an initial value. If we assume that $w(n-1)$, the maximal length of an $S_{n-1}$-killing sieve, is known, one can begin with $z \geq w(n-1)+1$ as $w(n) \geq w(n-1)+1$. If the initial choice of $z$ is too high, no $S_{n}$-killing sieve of length $z$ will be found. If an $S_{n}$-killing sieve of length $z$ is found, then the algorithm proceeds by finding an $S_{n}$-killing sieves of increasing length until there is a $z$ for which no $S_{n}$-killing sieve of length $z$ can be found. Then $w(n)=z-1$ and $h(n)=2 w(n)+2$.

The algorithm cycles through all possible $c \in \mathbf{Z}_{R}^{*}$, where $R=R_{n}(z)$ (unless $105 \leq z<111$ ). We note that as $z$ increases, there are fewer possible choices of $c$ and $c_{i}$ satisfying (4.1), (4.2) (see also the conditions in Steps 5 and 6 (iii) below). Hence, as long as $R$ remains unchanged, the search runs progressively faster as $z$ increases. If $z$ increases and $R$ changes, then the search slows down as the number of possible $c$ that need to be examined in Step 4 increases.

Finally, we note that when $z>R_{n}(z)$ (which only occurs when $z<70$ or $106 \leq z \leq 110)$, we can modify the algorithm by using the set $\{3,5,7,11\}$ in place of the set $S_{n}(z)$ and define $r=4, R=1155$ in lieu of $r_{n}(z), R_{n}(z)$.

\section{Example: Calculation of $h(20)$}

In this section, we work out the details of the algorithm in Table 4 for calculating $h(20)$. Suppose that we are looking for a gap of length $h(20)=174$, or equivalently, an $S_{19}$-killing sieve of length $z=86$. Then Definition 3.6 gives $S=S_{19}(z)=$ $\{3,5,7\}, R=R_{19}(z)=105$, and $r=r_{19}(z)=3$. Then $Z=\mathbb{Z}_{105}^{*}$. We will need

to search over the elements $c \in Z$ and the congruence classes for the primes in $S_{19}-S_{19}(z)=\{11,13, \ldots, 71\}$.

Suppose we have chosen $c=29 \in Z$ and let $c_{i} \equiv c \bmod q_{i}$, for $i=1,2,3$. Then $T_{0}=\{(1,2 \bmod 3),(2,4 \bmod 5),(3,1 \bmod 7)\}$ and $I_{3}=I_{3}\left(T_{0}, 86\right)$ is the 37-element set

$$
\begin{aligned}
& I_{3}=\{3,6,7,10,12,13,16,18,21,25,27,28,30,31,33,37,40,42,45, \\
&46,48,51,52,55,58,60,61,63,66,67,70,72,73,75,76,81,82\} .
\end{aligned}
$$


TABLE 2. Algorithm for calculating a maximal $S_{n}$-killing sieve. Starting with an initial value of $z \leq \omega(n)$, the algorithm finds $S_{n^{-}}$ killing sieves of increasing length $z . w(n)$ is the final $z$ for which an $\left(S_{n}, k\right)$-killing sieve of length $z$ can be successfully found.

1. Begin with a positive integer $z$.

2. If $z<111$, let $r=4, R=1155$. Otherwise, let $R=R_{n}(z), r=r_{n}(z)$ using Definition 3.6

3. Let $Z=\mathbb{Z}_{R}^{*}$.

4. Choose $c \in Z$. If every element of $Z$ has already been chosen, then there is no $S_{n}$-killing sieve of length $z$.

5. Let $I_{r}=[1, z] \cap\left(\mathbb{Z}_{R}^{*}+c\right)$, where $W+c$ is the set defined by adding $c$ to each element of a set $W$. If $\left|I_{r}\right|>M(n, w, r)$, then choose a different $c$ in Step 4 Otherwise, let $i_{1}=1, \ldots, i_{r}=r, T_{0}=\{(1, c), \ldots,(r, c)\}, h=0$, and $k_{0}=0$.

6 . If $h<n-r-k_{h}$, then:

a. Let $j$ be the smallest integer with $i_{r+h}+1 \leq j \leq n$ and the property that there exists $x, y \in I_{r+h}\left(T_{h}\right)$ with $x \equiv y \bmod q_{j}$.

b. If no such $j$ exists, then:

i. Let $k_{h}=n-r-h$. If $\left|I_{r+h}\right| \leq k_{h}$, then continue with Step 7 Otherwise, if $h=0$, continue with Step 4, if $h>0$, continue with Step 6r(i).

c. If $j$ exists, let $h=h+1, i_{r+h}=j$ and $k_{h}=k_{h-1}+j-i_{r+h-1}-1$. Then:

i. Choose $c_{r+h} \bmod q_{i_{r+h}}$. (If all possible choices for $c_{r+h} \bmod q_{i_{r+h}}$ have already been made (for a fixed $T_{h-1}$ ), then if $h \geq 2$, repeat this step with $h=h-1$. If $h=1$, let $h=0$ and continue with Step 4)

ii. Let $T_{h}=T_{h-1} \cup\left\{\left(i_{r+h}, c_{r+h}\right)\right\}$ and $I_{r+h}=I_{r+h}\left(T_{h}, z\right)$.

iii. If $\left|I_{r+h}\right| \leq k_{h}+M\left(n, z, i_{r+h}\right)$, continue with Step 6 Otherwise, go to Step 6 c(i).

7. $T_{h}$ determines an $\left(S_{n}, k_{h}\right)$-killing sieve of length $z$ with $r+h$ elements. Then: a. Increment $z$. Let $R_{0}=R$ and recalculate $r, R$ as in Step 2

b. If $R=R_{0}$, then continue with Step 4 and the same $c$. Subsequently, one need only consider $c \in Z$ not previously considered by the algorithm.

c. If $R \neq R_{0}$, let $\pi$ be the projection map $\pi: \mathbb{Z}_{R} \rightarrow \mathbb{Z}_{R_{0}}$. Let $Z^{\prime} \subset Z$ be the set consisting of $c$ and elements in $Z$ not previously considered by the algorithm. Then replace $Z$ by $\pi^{-1}\left(Z^{\prime}\right)$ and continue with Step 4

Using the notation of Step 5, we have $i_{1}=1, i_{2}=2, i_{3}=3$. Now $m(q, 86)=5$, for $q=11,13 ; m(q, 86)=4$, for $q=17,19 ; m(q, 86)=3$, for $q=23$; and $m(q, 86)=2$, for $q=29, \ldots, 71$. Hence, we have $M(19,86,3)=43 \geq 37=\left|I_{3}\right|$. As the bound given by (3.2) for $k=3$ is satisfied, it may be possible that $T_{0}$ can be extended to an $S_{19}$-killing sieve of length 86 .

We now start with Step 6 in Table 4 We have $h=k=0$ and $r=3$. Since the elements $3,25 \in T_{0}$ are congruent $\bmod 11$, we can take $h=1$ and $i_{4}=4$. If we choose $c_{4} \equiv 7 \bmod 11$, we have $T_{1}=T \cup\{(4,7 \bmod 11)\}$ and $I_{4}=I_{4}\left(T_{1}\right)=$ $I_{3}-\{7,18,40,51,73\}$. Then the bound given by (3.2) is satisfied for $k=4$ as $M(19,86,4)=38 \geq 32=\left|I_{4}\right|$. 
By the same logic, we can choose $h=2, i_{5}=5$, and $c_{5} \equiv 3 \bmod 13$. Then $I_{5}=I_{4}-\{3,16,42,55,81\}$ and $M(19,86,5)=33 \geq 27=\left|I_{5}\right|$. Continuing in this manner, we find that for $h=1,2, \ldots, 12$, there is a choice of $c_{3+h} \bmod q_{3+h}$ (given by the entries in the column $n=19$ in Table 3) such that the inequality $M(19,86, j) \geq\left|I_{j}\right|$ is satisfied. Then

$$
T_{12}=T_{0} \cup\left(\bigcup_{h=1}^{12}\left\{\left(i, c_{i} \bmod q_{i}\right)\right\}\right) .
$$

Proceeding through the algorithm, in Step [6, when $h=12$ and $k_{12}=0$, we find in Step 6 that no such $j$ exists. Hence in Step $6 \mathrm{~b}$ we set $k_{12}=4$. The algorithm then concludes that $T_{12}$ is an $\left(S_{19}, 4\right)$-killing sieve of length 86 .

We note that with the sieve $T_{12}$, there are five primes $(q=23,59,61,67,71)$, where $n_{i}$ (with $i$ determined by $q=q_{i}$ ) is less than the optimal bound $m\left(q_{i}, 86\right.$ ). In these cases, we have $n_{i}=m\left(q_{i}, 86\right)-1$. One might hope that a different choice of the $c_{i}$ would permit $n_{i}=m\left(q_{i}, 86\right)$ and result in a longer $S_{19}$-killing sieve.

Suppose we try to find an $S_{19}$-killing sieve of length 87 . Using the same data as above, we would again arrive at Step $6 \mathrm{~b}$ with $h=12$ and then choose $k_{12}=4$; but now $\left|I_{15}\right|=5>4=k_{12}$. Hence, there is no $S_{19}$-killing sieve of length 87 using this congruence data.

Finally, suppose in our original search for an $S_{19}$-killing sieve with length 86 , we had started with $c=97$. Then $\left|I_{3}\right|=40$. Picking $i_{4}=4, c_{4} \equiv 6 \bmod 11$, and $i_{5}=5, c_{5} \equiv 4 \bmod 13$, we have $n_{4}=n_{5}=2$ and $\left|I_{5}\right|=36$. But since $M(19,86,5)=33<\left|I_{5}\right|$, we know that there is no $S_{19}$-killing sieve associated with $c=97$ and $T_{2}=\{(1,97),(2,97),(3,97),(4,6),(5,4)\}$. Hence, in the algorithm, one must consider other congruence data for the primes $q_{4}, q_{5}$ and other $c \in Z$ to find an $S_{19}$-killing sieve of length 86 .

\section{Details of Computation}

The algorithm was coded in C, and initially run on a Linux 2.6 Ghz server. The program uses minimal memory, and processor speed is the main constraint for the calculation of $h(n)$ for increasing values of $n$. For $n=42$, the calculation took approximately two months (we note that once $h=h(n)$ is known, it is much faster to verify that $h(n)=h$ by starting the search with $z=h$ ). To enable the calculation of $h(n)$ for $n \geq 43$, a distributed computing approach was used [13] to enable a number of computers to simultaneously search different areas of the search space for $a \in Z$ (see Step 3 of the algorithm). With a cluster of thirty computers (GNU/Linux $2.4 \mathrm{Ghz}$ ), the calculation of $h(49)$ took approximately two months.

Let $w_{n-1}$ be the value calculated by the computer program as the maximal length of an $S_{n-1}$-killing sieve. From the data in Tables 3 and 4 , it is simple to verify that there is a killing sieve of length $w_{n-1}$. Hence $w(n-1) \geq w_{n-1}$, and the values in Table 1 provide lower bounds for $h(n)$. Assuming a correct implementation in the computer program of the algorithm in this paper, these lower bounds are the actual values of $w(n-1), h(n)$, respectively. We note that the computer program's calculations agree with previous calculations of $h(n)$, for $n \leq 24$. 
TABLE 3. Congruence data for an $S_{n}$-killing sieve of maximal length $w$ for $19 \leq n \leq 33$.

Explanation of Tables [3, 4. Fix $19 \leq n \leq 48$ and let $S_{n}=\left\{q_{1}, \ldots, q_{n}\right\}$, where $q_{i}$ is the $i$ th odd prime. Fix the table column corresponding to $n$. The shaded rows indicate the primes in $S_{n}(w(n))$ for that $n$. Let $R=$ $\left\{r_{1}, \ldots, r_{t}\right\} \subset S_{n}$ be the set of those primes for which the corresponding entry is an asterisk. For $q \notin R$, let $c_{q}$ be the corresponding entry. The set $T=\left\{\left(i, c_{q_{i}}\right) \mid\right.$ for $i$ such that $\left.q_{i} \notin R\right\}$ forms a $\left(S_{n}, t\right)$-killing sieve of length $w(n)$. Let $I=\left\{y_{1}, \ldots, y_{t}\right\} \subset[1, z]$ be the subset of $t$ integers associated to $T$. Choose $a \in \mathbb{Z}$ such that $a \equiv-c_{q} \bmod q$, for all primes $q \in S_{n} \backslash R$ and $a \equiv-y_{i} \bmod r_{i}$ for $i=1, \ldots, t$. The sequence $a+1, \ldots, a+w(n)$ has length $w(n)$ and every term is divisible by a prime in $S_{n}$. Finally, $2 a+1+\prod_{i=1}^{n} q_{n}$ is the first term of a sequence of $h(n+1)-1$ integers that are each divisible by one of the first $n+1$ primes.

\begin{tabular}{|c|c|c|c|c|c|c|c|c|c|c|c|c|c|c|}
\hline 19 & 20 & 21 & 22 & 23 & 24 & 25 & 26 & 27 & 28 & 29 & 30 & 31 & 32 & 33 \\
\hline 174 & 190 & 200 & 216 & 234 & 258 & 264 & 282 & 300 & 312 & 330 & 354 & 378 & 388 & 414 \\
\hline 86 & 94 & 99 & 107 & 116 & 128 & 131 & 140 & 149 & 155 & 164 & 176 & 188 & 193 & 206 \\
\hline 2 & 1 & 2 & 1 & 2 & 2 & 2 & 2 & 2 & 2 & 1 & 1 & 2 & 1 & 2 \\
\hline 4 & 2 & 4 & 2 & 4 & 2 & 4 & 4 & 3 & 4 & 3 & 3 & 1 & 2 & 3 \\
\hline 1 & 2 & 3 & 2 & 3 & 6 & 3 & 3 & 4 & 3 & 2 & 3 & 3 & 4 & 1 \\
\hline 7 & 4 & 4 & 4 & 1 & 7 & 4 & 4 & 9 & 4 & 3 & 9 & 4 & 1 & 7 \\
\hline 3 & 11 & 7 & 11 & 7 & 10 & 7 & 7 & 1 & 7 & 6 & 6 & 2 & 2 & 4 \\
\hline 10 & 3 & 8 & 3 & 6 & 13 & 8 & 8 & 2 & 8 & 7 & 14 & 5 & 16 & 11 \\
\hline 6 & 18 & 2 & 3 & 17 & 9 & 2 & 2 & 15 & 2 & 1 & 12 & 6 & 10 & 18 \\
\hline 14 & 14 & 13 & 10 & 12 & 1 & 13 & 13 & 7 & 13 & 12 & 12 & 10 & 21 & 21 \\
\hline 12 & 10 & 1 & 8 & 2 & 4 & 1 & 1 & 24 & 1 & 2 & 2 & 14 & 9 & 10 \\
\hline 21 & 4 & 27 & 6 & 25 & 15 & 27 & 27 & 22 & 28 & 26 & 10 & 7 & 5 & 19 \\
\hline 30 & 8 & 16 & 16 & 29 & 31 & 16 & 28 & 32 & 1 & 13 & 21 & 12 & 20 & 17 \\
\hline 31 & 33 & 22 & 39 & 21 & 17 & 18 & 16 & 12 & 18 & 17 & 10 & 19 & 26 & 31 \\
\hline 33 & 41 & 12 & 35 & 11 & 17 & 20 & 32 & 10 & 16 & 15 & 34 & 32 & 24 & 25 \\
\hline 28 & 6 & 28 & 36 & 5 & 16 & 28 & 6 & 6 & 12 & 11 & 15 & 18 & 5 & 8 \\
\hline 13 & $*$ & 43 & 21 & 42 & 3 & 6 & 43 & 37 & 43 & 5 & 11 & 11 & 15 & 52 \\
\hline$*$ & 21 & $*$ & 45 & 15 & 19 & 43 & 8 & 2 & 8 & 7 & 37 & 9 & 8 & 24 \\
\hline$*$ & 29 & 6 & 14 & 50 & 39 & 60 & 51 & 51 & 57 & 59 & 13 & 27 & 6 & 9 \\
\hline$*$ & * & $*$ & 29 & * & 54 & 51 & 63 & $*$ & * & 7 & 44 & 42 & 16 & 42 \\
\hline$*$ & $*$ & $*$ & * & * & 25 & 55 & 61 & 55 & 61 & $*$ & 19 & 26 & * & 29 \\
\hline & 5 & 18 & * & 17 & 21 & 57 & 60 & 54 & 60 & 56 & 29 & 26 & 65 & 34 \\
\hline & & $*$ & 5 & 26 & 45 & 12 & 12 & 57 & 63 & $*$ & 26 & 5 & 35 & 8 \\
\hline & & & 18 & $*$ & 43 & * & 55 & 49 & 55 & 54 & 54 & 55 & 51 & 61 \\
\hline & & & & 27 & $*$ & * & $*$ & $*$ & $*$ & 27 & 36 & 85 & 75 & 46 \\
\hline & & & & & * & * & * & 45 & 51 & 62 & 47 & 57 & 59 & 27 \\
\hline & & & & & & 22 & 22 & 16 & 22 & 21 & 30 & 34 & 69 & 76 \\
\hline & & & & & & & 18 & 21 & 27 & 29 & 56 & 30 & * & 12 \\
\hline & & & & & & & & $*$ & * & * & 39 & 58 & 66 & 49 \\
\hline & & & & & & & & & * & $*$ & 5 & 78 & 71 & 66 \\
\hline & & & & & & & & & & 42 & 27 & 40 & 63 & 16 \\
\hline & & & & & & & & & & & $*$ & $*$ & 14 & 60 \\
\hline & & & & & & & & & & & & 13 & 30 & $*$ \\
\hline & & & & & & & & & & & & & 3 & * \\
\hline & & & & & & & & & & & & & & 6 \\
\hline
\end{tabular}


TABLE 4. Congruence data for an $S_{n}$-killing sieve of maximal length $w$ for $34 \leq n \leq 48$.

\begin{tabular}{|c|c|c|c|c|c|c|c|c|c|c|c|c|c|c|c|}
\hline$n$ & 34 & 35 & 36 & 37 & 38 & 39 & 40 & 41 & 42 & 43 & 44 & 45 & 46 & 47 & 48 \\
\hline$h(n+1)$ & 432 & 450 & 476 & 492 & 510 & 538 & 550 & 574 & 600 & 616 & 642 & 660 & 686 & 718 & 742 \\
\hline$w(n)$ & 215 & 224 & 237 & 245 & 254 & 268 & 274 & 286 & 299 & 307 & 320 & 328 & 342 & 358 & 370 \\
\hline 3 & 1 & 1 & 2 & 2 & 1 & 1 & 1 & 1 & 1 & 1 & 1 & 1 & 2 & 1 & 1 \\
\hline 5 & 3 & 1 & 4 & 4 & 3 & 1 & 1 & 1 & 2 & 1 & 2 & 4 & 4 & 1 & 3 \\
\hline 7 & 5 & 2 & 1 & 6 & 5 & 5 & 3 & 5 & 1 & 3 & 4 & 5 & 5 & 4 & 2 \\
\hline 11 & 6 & 2 & 6 & 7 & 6 & 10 & 5 & 5 & 6 & 10 & 1 & 7 & 5 & 1 & 7 \\
\hline 13 & 4 & 8 & 12 & 5 & 4 & 5 & 10 & 5 & 10 & 1 & 8 & 1 & 3 & 2 & 11 \\
\hline 17 & 9 & 3 & 15 & 3 & 3 & 8 & 1 & 13 & 8 & 16 & 3 & 4 & 6 & 14 & 9 \\
\hline 19 & 8 & 7 & 1 & 9 & 5 & 14 & 3 & 14 & 13 & 9 & 14 & 6 & 7 & 14 & 14 \\
\hline 23 & 5 & 4 & 17 & 10 & 9 & 4 & 21 & 3 & 12 & 21 & 11 & 15 & 2 & 5 & 13 \\
\hline 29 & 3 & 5 & 2 & 17 & 16 & 15 & 20 & 5 & 11 & 12 & 6 & 6 & 20 & 24 & 12 \\
\hline 31 & 11 & 27 & 11 & 12 & 11 & 12 & 12 & 12 & 3 & 24 & 5 & 11 & 21 & 7 & 4 \\
\hline 37 & 7 & 32 & 13 & 30 & 14 & 2 & 15 & 8 & 16 & 3 & 9 & 7 & 9 & 34 & 17 \\
\hline 41 & 21 & 39 & 11 & 32 & 25 & 2 & 2 & 2 & 4 & 11 & 14 & 24 & 31 & 10 & 21 \\
\hline 43 & 35 & 24 & 16 & 29 & 34 & 20 & 9 & 5 & 41 & 9 & 41 & 40 & 24 & 20 & 10 \\
\hline 47 & 20 & 6 & 29 & 36 & 43 & 38 & 8 & 14 & 33 & 8 & 18 & 30 & 36 & 10 & 22 \\
\hline 53 & 34 & 45 & 27 & 16 & 34 & 23 & 30 & 6 & 21 & 30 & 13 & 37 & 5 & 29 & 22 \\
\hline 59 & 14 & 18 & 38 & 45 & 32 & 48 & 33 & 40 & 9 & 48 & 16 & 23 & 28 & 3 & 15 \\
\hline 61 & 15 & 52 & 14 & 42 & 57 & 1 & 12 & 1 & 5 & 15 & 49 & 3 & 15 & 30 & 26 \\
\hline 67 & 59 & 62 & 21 & * & 44 & 9 & 63 & 14 & 60 & 39 & 1 & 20 & 1 & 47 & 6 \\
\hline 71 & 58 & 33 & 10 & 50 & 21 & 24 & 48 & 69 & 56 & 57 & 26 & 45 & 14 & 17 & 10 \\
\hline 73 & 28 & 14 & 67 & 47 & 35 & 35 & 37 & 51 & 54 & 1 & 15 & 60 & 22 & 37 & 47 \\
\hline 79 & 7 & 38 & 33 & 21 & 7 & * & 65 & 35 & 48 & 42 & 29 & 7 & 70 & 8 & 18 \\
\hline 83 & 24 & 75 & 82 & 44 & 1 & 17 & 14 & 24 & 44 & 29 & 67 & 2 & 5 & 59 & 42 \\
\hline 89 & 36 & 48 & 2 & 58 & 13 & 25 & 39 & 17 & 38 & 78 & 12 & 78 & 73 & 79 & 42 \\
\hline 97 & 2 & 95 & 14 & 60 & 80 & 80 & 55 & 65 & 30 & 13 & 46 & 1 & 30 & 9 & 7 \\
\hline 101 & 66 & 7 & 7 & 25 & 99 & 69 & 57 & 42 & 26 & 2 & 44 & 71 & 13 & 72 & 88 \\
\hline 103 & 92 & 12 & 18 & 5 & 41 & 52 & 32 & 50 & 24 & 62 & 50 & 8 & 20 & 35 & 92 \\
\hline 107 & 102 & 78 & 70 & 61 & 7 & 30 & 40 & 102 & 20 & 18 & 94 & 49 & 106 & 88 & 8 \\
\hline 109 & 41 & 74 & 48 & 87 & 65 & 53 & 53 & 20 & 22 & 46 & 30 & 28 & 38 & 105 & 20 \\
\hline 113 & 57 & 42 & 37 & 52 & 16 & 45 & 19 & 29 & 10 & 19 & 63 & 49 & 37 & * & 5 \\
\hline 127 & 29 & 15 & 3 & 81 & * & 13 & 50 & 113 & 1 & 35 & 19 & 80 & 63 & 107 & 119 \\
\hline 131 & 45 & 84 & * & 112 & 15 & 3 & 99 & 13 & 28 & 63 & 52 & 102 & 10 & 129 & 80 \\
\hline 137 & * & 15 & 55 & 1 & 27 & 72 & 72 & 87 & 129 & 75 & 114 & 48 & 14 & 27 & 132 \\
\hline 139 & 71 & 29 & * & 93 & 2 & 29 & 29 & 80 & 125 & 119 & 59 & 113 & 44 & 83 & 7 \\
\hline 149 & * & * & 46 & 82 & 36 & 78 & 123 & 78 & 135 & $*$ & 141 & 132 & 97 & 84 & 22 \\
\hline 151 & & 59 & 45 & 15 & 59 & 104 & 68 & 77 & 143 & 23 & 38 & 146 & 132 & 113 & 4 \\
\hline 157 & & & 30 & 78 & * & 92 & 47 & 32 & 119 & 20 & 131 & 41 & 18 & 125 & 122 \\
\hline 163 & & & & T & 29 & 77 & 77 & * & 95 & 5 & 140 & 50 & 90 & 77 & 1 \\
\hline 167 & & & & & 60 & * & * & 15 & 111 & 72 & 69 & 36 & 100 & 42 & 57 \\
\hline 173 & & & & & & 60 & 42 & 39 & 18 & 60 & $*$ & 57 & 160 & 117 & 27 \\
\hline 179 & & & & & & & 54 & 9 & * & $*$ & 51 & * & 118 & 153 & 126 \\
\hline 181 & & & & & & & & $*$ & $*$ & 68 & 104 & 56 & 105 & 98 & 56 \\
\hline 191 & & & & & & & & & 63 & 69 & 24 & $*$ & 112 & * & * \\
\hline 193 & & & & & & & & & & 89 & 113 & 17 & * & 44 & 137 \\
\hline 197 & & & & & & & & & & & 48 & 108 & 43 & 75 & 39 \\
\hline 199 & & & & & & & & & & & & 32 & 66 & 68 & 101 \\
\hline 211 & & & & & & & & & & & & & 51 & 134 & 110 \\
\hline 223 & & & & & & & & & & & & & & 50 & 134 \\
\hline 227 & & & & & & & & & & & & & & & 45 \\
\hline
\end{tabular}




\section{ACKNOWLEDGMENTS}

The author wishes to express his thanks to R. Murty and Q. Zheng for introducing him to the Jacobsthal function, to J. Haugland for explaining his computational work on $h(n)$, to M. Pulimood and her students for their work in grid computing that enabled the calculation of the larger values of $h(n)$, and to M. Kobayashi and the referee for their helpful comments and careful reading of this manuscript. The author also wishes to thank The College of New Jersey for a SOSA award that supported this work.

\section{REFERENCES}

[1] H. Cramér, On the order of magnitude of the difference between consecutive prime numbers, Acta. Arith. 2, 396-403, (1936).

[2] P. Erdös, On the integers relatively prime to $n$ and on a number theoretic function considered by Jacobsthal, Math. Scand., 10, 163-170, (1962). MR0146125(26:3651)

[3] D. Gordon, G. Rodemich, Dense Admissible Sets, In: Algorithmic Number Theory, Lecture Notes in Computer Science 1423, Springer-Verlag, Berlin, 1998. MR1726073 (2000k:11104)

[4] J. Haugland, private correspondence, July 2005.

[5] H. Iwaniec, On the error term in the linear sieve, Acta. Arith. 19, 1-30, (1971). MR0296043 $(45: 5104)$

[6] H. Iwaniec, On the problem of Jacobsthal, Demonstratio Math. 11, 225-231, (1978). MR 499895 (80h:10053)

[7] E. Jacobsthal, Uber Sequenzen ganzer Zahlen, von denen keine zu $n$ teilerfremd ist. I-III, Norske Vid. Selsk. Forhdl. 33, 117-124, 125-131, 132-139, (1960). MR0125047 (23:A2354)

[8] N.J.A. Sloane, (2007), The On-Line Encyclopedia of Integer Sequences, published electronically at http://www.research.att.com/〜njas/sequences/, Sequence A048670.

[9] H.J. Kanold, Über eine zahlentheoretische Funktion von Jacobsthal, Math. Annalen 170, 314-326 (1967). MR0209247 (35:149)

[10] H.J. Kanold, Neuere Untersuchungen über die Jacobsthal-Funktion g(n), Monatsh. Math., 84, 109-124 (1977). MR0453672(56:11932)

[11] H. Maier, C. Pomerance, Unusually Large Gaps Between Consecutive Primes, Trans. Amer. Math. Soc. 322(1), 201-237, (1990). MR972703 (91b:11093)

[12] J. Pintz, Very large gaps between consecutive primes, J. Number Theory, 63(2), 286-301, (1997). MR1443763(98c:11092)

[13] S.M. Pulimood, and T.R. Hagedorn, Applying the Grid Computing Paradigm within a Liberal Arts Academic Environment. In Proceedings of the 2007 International Conference on Grid Computing Applications (GCA 2007) held in conjunction with The 2007 World Congress in Computer Science, Computer Engineering, and Applied Computing, Las Vegas, USA, June 2007.

[14] R. Rankin, The difference between consecutive prime numbers. V, Proc. Edinb. Math. Soc. (2) 13, 331-332, (1962/63). MR0160767 (28:3978)

[15] H. Stevens, On Jacobsthal's g(n)-function, Math. Annalen, 226, 95-97, (1977). MR0427212 $(55: 247)$

Department of Mathematics and Statistics, The College of New Jersey. P.O. Box 7718, Ewing, New JerSey 08628-0718

E-mail address: hagedorn@tcnj.edu 